\title{
Hybrid Framework for a Robust Face Recognition System Using EVB_CNN
}

\author{
Tamilselvi M., Sathyabama Institute of Science and Technology, Chennai, India
}

S. Karthikeyan, Sathyabama Institute of Science and Technology, Chennai, India

\begin{abstract}
Recognition of the human face is becoming an ingenious technology that enhancing its strategy gradually by finding its applications in a wide variety of fields including security and surveillance. The traditional methods that are in practise for face recognition are not adequate in producing good accuracy due to two main reasons. The first one is the pictures are affected by various uncontrolled situations such as illumination, blur, and pose, and the second one is struggling in an efficient recognition when dealing with a large number of samples. There is need for an effective face recognition as a part of life in the automated environment. The traditional methods are lagging with some parameters. To overcome the aforementioned issues, a new methodology is implemented. This methodology is a hybrid frame work combined with Eigen value-based convolutional neural networks (EVB_CNN). The EVB_CNN is designed in such a way that the significant features are extracted and classified by the softmax function and fully connected layer, respectively. The experimental analysis is carried out with AR data set and ORL data set that shows enhancement in accuracy with significant reduction in computation time with images taken over specific uncontrolled environments.
\end{abstract}

\section{KEYWORDS}

Biometrics, CNN, Face Detection, Face Recognition, Feature Extraction, Softmax Function

\section{INTRODUCTION}

In a vision structure, human images are more static over the person's life time. Hence identifying or recognizing images are not surprising capability for human being. At the same time, Yang et al (2017) states that it needs a glance of person's face to think back about the particular person. Human brain is having particular part exclusively to identify and remember the images by recalling the key details of the face like nose, eyes, ears, mouth and cheeks. Ramkumar et al (2018) states that, the human brain can recognize the faces to some extent even the image has been affected by uncontrolled situations such as illumination, blur and pose variation. On the opposite side Yan et al (2017) mentioned that face recognition by computer is depends upon the predefined factors. If the face is affected by the fore said issues, then the data needed for a computer to recognize the face is increasing and the computing operations are also becoming tricky. In this regard, Yuan et al (2017) discuss that many machine learning algorithms were developed that includes Deep Neural Network, CNN, Deep Belief Network (DBN) and Artificial Intelligence (AI). Since, enormous development in face recognition

\section{DOI: 10.4018/JCIT.20210701.oa4}

This article, published as an Open Access article on April 23, 2021 in the gold Open Access journal, Journal of Cases on Information Technology (JCIT) (converted to gold Open Access January 1, 2021), is distributed under the terms of the Creative Commons Attribution License (http://creativecommons.org/licenses/by/4.0/) which permits unrestricted use, distribution, and production in any medium, provided the author of the original work and original publication source are properly credited. 
technology gradually increasing the purpose of interacting with humans and recognition of face finds major part in many applications, Coskun et al (2017). Even though, there are many methods available for serving this purpose, we are in need of achieving more effective recognition with good accuracy and less computation time. That's the reason, How the face recognition technology become a wide area of research that grabs the creative ideas of the researchers, Itqan et al (2016).

Tamil et al (2019) states that traditional algorithms which are existing for face recognition are not handling the images effectively and also they could not process the large sample size of data which is a highly needed requirement in the current scenario with the known thing that the entire globe is moving gradually towards the automation to implicate the various purposes of recognizing faces to serve wide variety of applications including bio metric identification, serving security applications, monitoring and tracking live stream video etc.

Topical developments in the field of automatic face recognition, design acknowledgment and AI have made it conceivable to create an effective face recognition method to overcome the issues faced in various aspects of face recognition, Wang et al (2017). From one viewpoint, perceiving face is common procedure, since most of the individuals can do it easily with less cognizant. Then again, doing the same recognition with the help of machine is a troublesome issue. There are many traditional algorithms are available for face recognition. Generally, face recognition is done by putting the face recognition algorithms on two broad categories. They are holistic approach and local feature approach, Tian et al (2018).

The appearance-based methods are generally linear in nature and coming under the category of holistic approach that includes principal component analysis (PCA), independent component analysis (ICA), linear discriminate analysis (LDA) etc, Rajesh et al (2020), Shen et al (2018). These methods are not sufficient to face the images that are affected by poor lighting since the most important key features of the images are available over the non-linear plane. Highlighting these issues for an effective face recognition, the main objective of this work is arrived that implies a hybrid frame work of EVB_CNN for an effective face recognition over uncontrolled conditions with enhanced accuracy and less computation time. In the proposed method, the face recognition is performed in two important steps. (i)Detecting and normalizing the face using the calculated Eigen values (ii)Face recognition by $\mathrm{CNN}$ where the feature extraction and classification done as a combined work. Finally, afore mentioned steps effectively recognizing the face and showing enhancement in accuracy rate.

The remaining part of this paper is structured as like this II Theoretical Framework, III Literature survey, IV Methodology, V Sample Selection, Related work, VI Findings and Discussion VII Conclusion and Recommendation.

\section{THEORETICAL FRAMEWORK}

As mentioned in section I, the images taken over some unconfined situations are becoming the challenging task for the researchers to recognize the appropriate image in an accurate manner. Hence, in our proposed work we have taken the objective as to develop an efficient face recognition model for recognizing and processing the images taken over some unconfined situations. In our work, initially the samples are taken from an either AR and ORL data base and then they are resized according to the face region present in that frame. Then cropped images are pre-processed using Gabor filter in order to the noises from the image. Gabor filter is chosen for the reason that they are smooth enough to handle the images with non-linear variations. After the pre-processing, the images are further reduced in dimension by calculating the eigen value among the neighbour pixels there by detecting the face region and non-face region. Now the image is resized to 150x150 and contains only the face region. This samples are then trained by CNN where usually the dimension reduction is done at the softmax or in pooling layer which takes much time for computation. In our work, we are giving the cropped resized samples to the $\mathrm{CNN}$ for further operations such as feature extraction and classification that 
in turn reduces the computation time to a significant amount without comprising the accuracy. The proposed method and the experimental analysis are explained in the upcoming sections in detail.

\section{LITERATURE REVIEW}

Hein et al (2019) explained the significance of processing the images captured by indoor cameras where the lighting conditions are not good. They also state the problem of recognizing the human body along with face, where we are not concentrating much about the frontal view of face. In order to address these issues, they used multi modal human recognition method which concentrating on both body and face when captured by indoor cameras which are used for surveillance environments. Initially they detected the face by adaboost along with Harr classifier and then this has been given to CNN architecture as input. Here two separate CNN's are used for face and body respectively to extract the details in more accurate manner to improve the accuracy of the face recognition. They highlighted the uniqueness of their proposed method as, the importance of recognizing the unseen part of the body which is not taken in to account in the previously available methods. They took VGG Face-16 and ResNet-50 models for the experimentation and they have shown the equal error rates as $1.52 \%$ and $0.58 \%$ for Dongguk Face and body data base(DFB-DBI) and the open choke point database consequently.

Ramesh et al (2019) explained about the CNN based face recognition where CNN model is composed of 8 layer structure. They used stochastic gradient descent algorithm for extracting the feature and classification and the problem of over fitting is solved by Drop out method. For training and testing process, they used the frame work of convolution architecture which is for feature extraction. In this work, ORL and AR database are used and accuracy rate of $99.82 \%$ and $99.78 \%$ achieved consequently.

Jing Li et al (2018) stated about a new method called C2D-CNN (color 2- dimensional Principal Component Analysis Convolutional Neural Network), which is preferred to use for face recognition when there is lot of differences among training and testing category of images. They designed an algorithm which is used to combine the original image feature and the features extracted by the CNN. The proposed $\mathrm{CNN}$ is a model where the normalization layer is introduced to increase the recognition speed and to reduce the time required for training. At last, max-pooling layer is used to extract the maximum features. They used FRGC uncontrolled image and LFW data base. They justified that by their proposed method, the enhanced accuracy is achieved in face recognition.

Ji-hae kim et al (2017) invented a new algorithm to predict the facial expression based face recognition. They proposed an algorithm for facial expression as a combined combination of appearance and geometric features. In this work, initially they pre-processed the image using LBP and after this appearance feature model is applied to with draw the aggregate key points and the geometric model is used to pull out the significant land marks. Finally, that two models are combined and compared to effectively recognize the face expressions. They used CK+ and JAFEE data sets and shown accuracy rate of $96.5 \%$ and $91.3 \%$ consequently.

Koo J H et al (2018) stated the advantage of using convolutional neural network(CNN) for face recognition and also they compared the recognition rate with traditional methods like PCA and k-nearest neighbour (KNN). Here they used 7-layer model of CNN where two convolutional layers are used to form the feature maps. In this proposed method of CNN based face recognition, they shown $98.3 \%$ accuracy which is significantly more than PCA and KNN.

Elmahmudi Ali \& Ugail Hassan (2019) proposed a method to recognize the face in real time ie. Taken during capturing from camera or from video with effect less of the conditions like illumination, position and resolution. They named this method as HOG CNN to impart the significance of deep neural network in the area of face recognition. The HOG CNN is recognizing the face by the distance calculated from the captured image and the gallery image. 
Minghui Zhang, Yumeng Liang and Huadong Ma (2019) proposed a method of recognizing the emotion using CNN from the aware of context. First using RPN (Regional proposal network), the context is detected and then by calculalting the Euclidian distance. These inputs are given to CNN to recognize the emotion. EMOTIC data set is used for experimentation and enhanced performance is shown.

Dong et al (2018) introduced a combined method of CNN and SVM for effective face recognition which in turn shown good enhancement in face recognition and also in recognition rate. The CNN model is used to extract the features completely and then this has been classified by using the SVM (Support Vector Machine). The experimental results are explained with Casia web faces data bases and FERET data base. With this proposed method, $97.50 \%$ accuracy and 28(s) recognition rate is achieved.

Jiayu Dong, Huicheng Zheng and Lina Lian (2018) explained about the demanding issues with significance uses of face recognition. They proposed a CNN based model for FER (Face Expression Recognition) automatically. They also explained about the performance degradation of deep neural network with insufficient training data in small data bases used for expression. Hence, they introduced a new method of $\mathrm{CNN}$ along with dense connection for FER. They replaced the transition layers which has been used for down sample feature map with dense connectivity in pooling layers. $\mathrm{CK}+$ and OuluCASIA data bases are used for experimentation and analysis which ensures enhanced performance.

Behera Ardhendu and Andrew G.Gidney (2019) stated the importance of monitoring the head position recognition which finds number of applications in real time. They chosen this recognition of head position because of considering this as human behaviour indicator. The proposed method which traverse the image region in order to focus on the viewing point changes. HOG classifier is used to extract features. For experimentation open face 2.0 facial behaviour analysis tool kit and VGG face 2 data sets are used.

\section{METHODOLOGY}

The various issues that are faced in face recognition was discussed in detail in the previous section. In order to overcome the aforementioned challenges, we proposed a new method that recognizes the images effectively over the unconfined situations. In the existing CNN methods, the reconstruction of images is done at either the pooling layer or softmax layer. So it consumes much time to construct and train the network. Having said that, in our work we proposed EVB_CNN where the image is reconstructed in the pre-processing stage and the dimension is reduced to the size 150x150 and further this reconstructed images are trained with $\mathrm{CNN}$ that in turn reduces the computation time. On the other hand, the local feature approach is easily adaptable to the changes made on the images locally by some unconfined situations such as illumination, blur and pose. Hence in our work, we have chosen Gabor phase pattern to perceive the local changes and to protect them within the samples. Then the specific variations are identified by the eigen values of primary components of a face. The Eigen values are calculated for the probe and gallery faces and are compared with maximum likelihood features to remove the non-face region thereby reducing the dimension of the image.

This resized images are then trained by CNN to effectively extract the features and to classify the same. In the proposed method, we opted the $\mathrm{CNN}$ due to the reason that it is robust on handling the non-linear variations on the image. The main significance of our work is to reduce the computation time than the traditional methods by reducing the dimension before giving them to $\mathrm{CNN}$ for training in back propagation. Based on the structure, activation feature map is generated in softmax layer. Finally, the structured activation maps are going through the fully connected dense layers and the final classification has been done through which the images are recognized. This effort will give the enhancement in accuracy and this has been justified with samples taken from the AR and ORL data sets. These two data sets are consisting of various images that are affected due to the unconfined situations that helps to analyse the recognition. Finally, the error rates are taken with various values of 
epochs sets and they are compared to formulate the misclassification error rate. The figure 1 explains about the block diagram of proposed method.

\section{Preprocessing}

In the proposed work, pre-processing is done with Gabor filter which is good at extracting the element details in an efficient manner and also finding many applications in these research areas. The Gabor filter is chosen in this work since it is robust enough on local variations such poor lighting and expression variations. This Gabor filter is linear in nature and finds application in Image texture representation as like visual structure of human.

Two dimensional Gabor filter can be generalized as a function by the following equation:

$\mathrm{f}\left(\mathrm{x}, \mathrm{y}, \omega, \theta, \sigma_{\mathrm{x}}, \sigma_{\mathrm{y}}\right)=\left(1 / 2 \pi \sigma_{\mathrm{x}} \sigma_{\mathrm{y}}\right) \exp \left[\frac{-1}{2}\left(\left(\frac{x}{\sigma_{x}}\right)^{2}+\left(\frac{y}{\sigma_{y}}\right)^{2}\right)+j \omega(x \cos \theta+y \sin \theta)\right]$

In the above equation, Gabor filter can be considered as two dimensional function which is obtained by multiplying with Gaussian function.

The equation consists of the following parameters:

$\omega$ - central frequency

$\theta-$ Angle of orientation

$\sigma-$ spatial spread function

In frequency domain the function will be given as:

$\Psi(\mathrm{u}, \mathrm{v})={ }_{\mathrm{e}} \pi^{2} / \mathrm{f}^{2}\left(\gamma^{2}\left(\mathrm{u}^{\prime}-\mathrm{f}\right)^{2}+\eta^{2} \mathrm{v}^{12}\right)$

$\mathrm{u}^{\prime}=\mathrm{u} \cos \theta+\mathrm{v} \sin \theta$

$v^{\prime}=-u \sin \theta+v \cos \theta$

Figure 1. Block diagram of EVB_CNN model

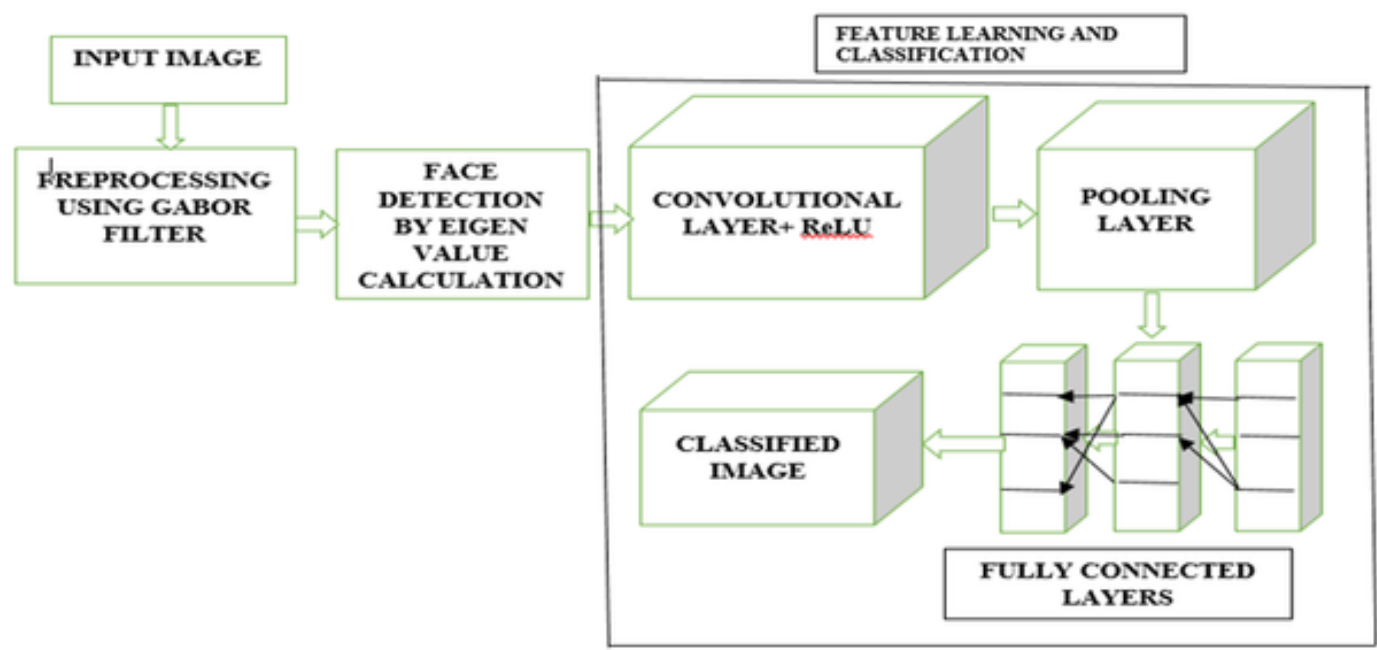


Figure 2 describes the Gabor filtered image. Gabor filters are also can be considered as Band pass filters which extends the band of frequencies. This filter gives us the information about the image both in frequency and spatial domain. It is used to detect the edges in the images so that the region of interest can be found for face detection. In our work, five scales and eight orientations has been applied.

Texture of image can be understood like which contains evaluated element details of the picture. This representation gives us the understanding of how the spatial domain coordinates are arranged and also about pixel element intensities. This texture image representation is very significant part in extraction and classification of images for face recognition.

\section{Calculation of Eigen Value}

In the preprocessing stage, the images are resized and the noises are removed. The images are now with the resized dimension of $150 \times 150$ that is then processed to calculate Eigen values by calculating the Euclidean distance among the neighbour pixels. This Eigen value explains the significance of this extracted specific value to reconstruct the image. It is also used in extracting the normalized features from images to ensure the image sharpness.

To calculate Eigen values of the images, the normalization is given by:

$$
(\mathrm{x}, \mathrm{y})=\frac{g(x, y)}{\sqrt{\sum_{x=0}^{N-1} \sum_{0}^{N-1}[g(x, y)]^{2}}}
$$

Mean operator computation is given by:

$\mu=\frac{1}{N^{2}} \sum_{x=0}^{N-1} \sum_{x=0}^{N-1} g(x, y)$

\section{Figure 2. Gabor filtered image}
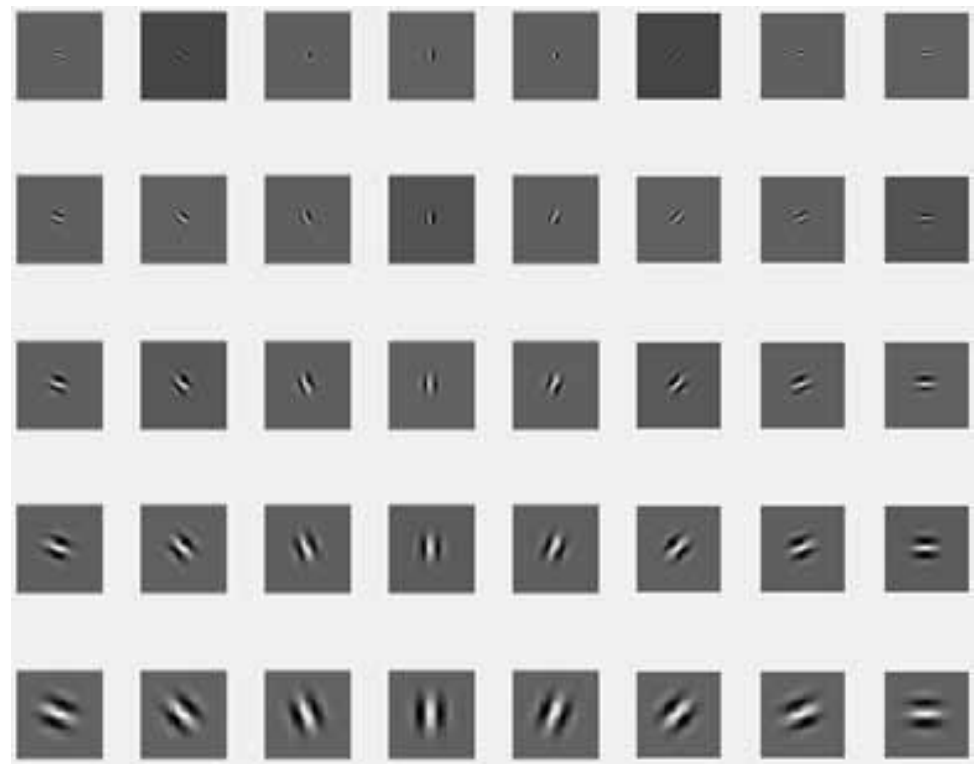
Covariance matrix is given by:

$\mathrm{S}_{\mathrm{g}}=\frac{1}{(N-1)} \mathrm{GG}^{\mathrm{T}}$

where $\mathrm{G}$ is matrix of $\mathrm{g}(\mathrm{x}, \mathrm{y})$ after removal of mean $(\mathrm{G}=\mathrm{G}-\mu)$. After calculating the Eigen values, except the region of interest, all other parts of the images or all other images are removed ie., faces and non faces regions are identified and cropped the face regions to reduce the dimension further more. The calculated weighted values detecting the faces in the images. After detecting the face, they are given to the $\mathrm{CNN}$ architecture to train the samples for feature extraction and classification to recognize the faces.

\section{Convolutional Neural Network (CNN) Model Using Tensor Flow}

In EVB_CNN, the convolutional neural networks are nonlinear models that can extract the features at the maximum when compared to the traditional methods such as LBP, DBC, SURF and SIFT. Here EVB_CNN is designed in such a way that the architecture consists of an input layer, hidden layers and an output layer. The hidden layer consists of various layers such as pooling layer, soft max. layer and fully connected layers. The CNN network trains these data to extract the key factors that are needed for an effective face recognition. In our work, the proposed CNN is modelled using tensor flow that is an upcoming artificial intelligence frame work of Google which is available as an open source and also it is easily run on many devices with good performance. The practical approach of our work is carried out with the face data sets such as AR and ORL. The experimental analysis is done with the various values of epochs taken for consideration to calculate the error rates and to find the misclassification rate. The Figure 3 implies the pictorial depiction of facial recognition process. Each layer is explained in detail in the following sub sections.

\section{Convolutional Layer}

The output from the input layer is given to the convolutional layer as input where the image details are represented in terms of matrices. The convolution matrix size chosen as $5 \times 5$. The feature map is created in this layer.

Figure 3. Pictorial depiction of facial recognition process

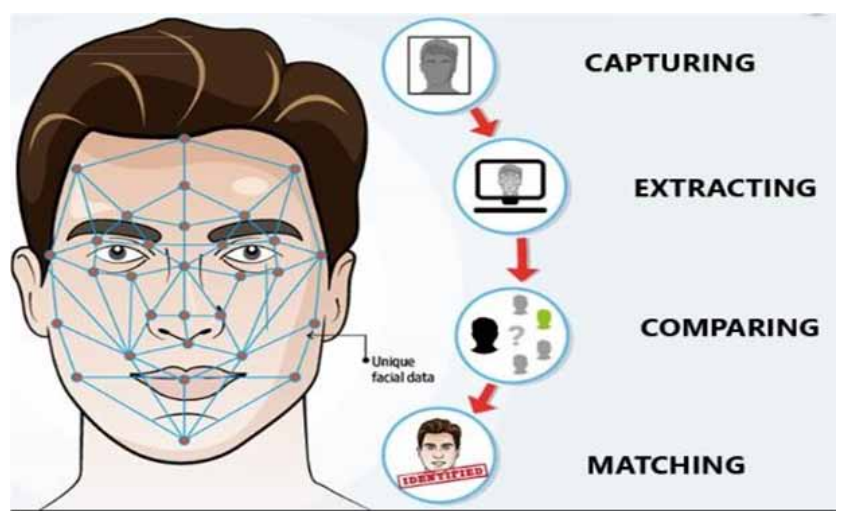


The convolutional layer is mathematically given as:

$$
\mathrm{x}_{\mathrm{j}}^{1}=\mathrm{f}\left(\sum_{i \in M_{j}^{l-1}} x_{i}^{l-1} k_{i j}^{l}+b_{j}^{l}\right)
$$

where:

$$
\begin{aligned}
& \text { l- layer } \\
& \mathrm{k}-\text { Kernel of convolution } \\
& \mathrm{b}-\text { bias } \\
& \mathrm{M}_{\mathrm{j}}-\text { Feature map }
\end{aligned}
$$

\section{Pooling Layer}

The calculated feature maps from the convolution layer are not good in dimension reduction which requires more time of computation. Hence, this layer is used in the reduction of dimension since it is nonlinear in nature. Figure 4(a) shows the maximum pooling layer and 4(b) shows the extraction from maximum pooling layer.

This pooling layer is involving in reducing size which means the feature map numbers are remaining unchanged instead it reduces the size of the feature map. It combines the neighbouring pixels into single pixel to reduce the dimension of the images. Mainly the convolution operation is performed in two-dimensional plane which is not in other neural network. It performs Maximum and minimum pooling.

\section{Fully Connected Layer}

In a CNN model, we have sequence of convolutional, softmax, pooling layers as a rack. Hence in order to connect all layers, some number of fully connected layers are placed before the output layer. This layer is responsible for image classification.

Mathematically it is expressed as:

$\mathrm{Y}_{\mathrm{pj}}=\mathrm{f}\left(\sum_{i=1}^{n} x_{i}^{l-1} w_{j i}^{l}+b_{j}^{l}\right)$

where:

$\mathrm{n}-$ neurons number in the previous layer

$\mathrm{f}$ - activation function of layer

\section{Softmax Layer}

This layer is nonlinear in nature hence it is used to model a more complex system for efficiently identify an image since the face is having more complex characteristics. This layer is serving the classification purpose.

The proposed CNN model can be explained with the following steps:

Step 1: The input images for which Eigen values are calculated and based on that region of interest are detected. 
Figure 4. (a) Max- pooling layer diagram; (b)Extraction from Max. pooling layer

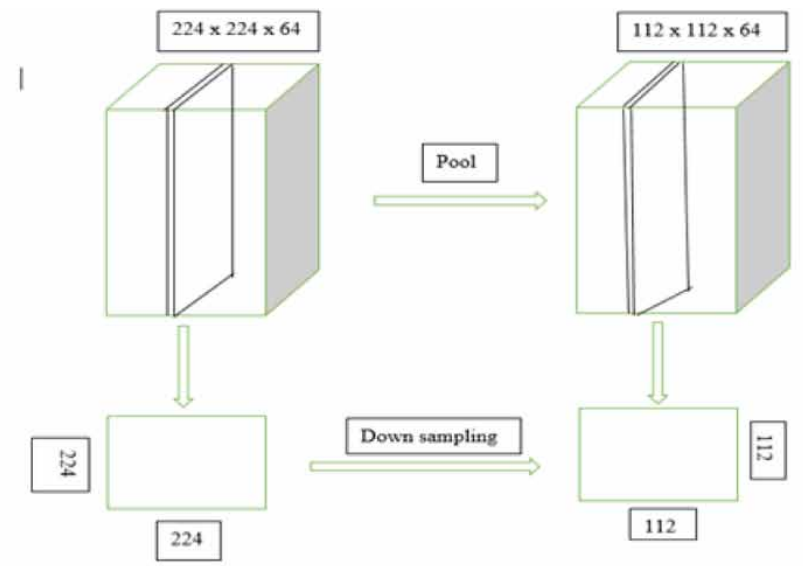

(a)

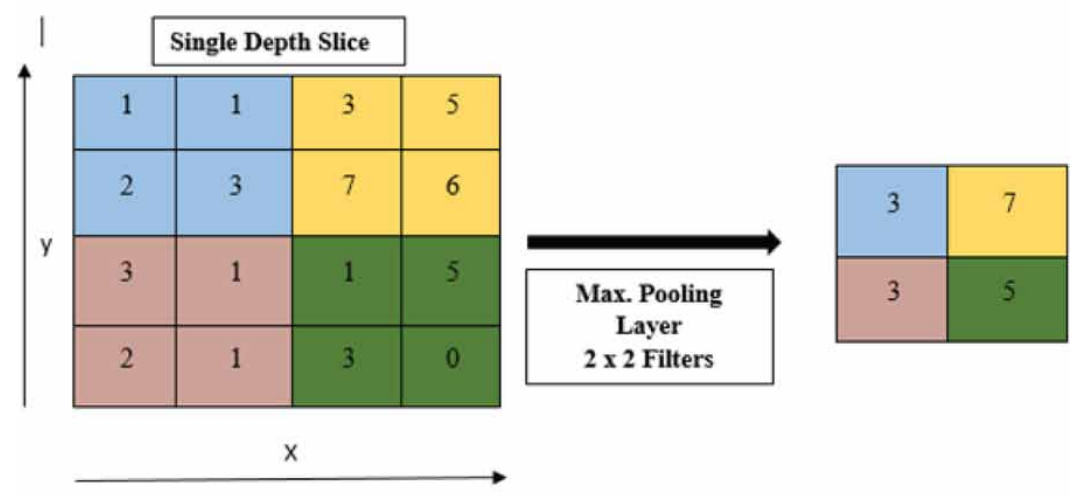

(b)

Step 2: Create a mat file as Conv.m to convert the image into convolution operation. ie., converting the image into matrix structure

Step 3: Create a pool.m file to process the combination of convolution layer. It reduces the dimension by combining the neighbouring pixels into single pixel. Mainly the convolution operation is performed in two dimensional plane which is not in other neural network. It performs maximum and minimum pooling.

Step 4: The convolutional layer generating the feature map for the images. The number of convolutional layers are same as number of featured map. Since the convolutional layer filters are 2D matrices, they are send to training process.

Step 5: Formulating ReLu and softmax activation function in between convolutional layer and feature map.

Step 6: Three weight matrices $\mathrm{w} 1, \mathrm{w} 5, \mathrm{w} 0$ are created for feature maps where $\mathrm{w} 1$ is the weight of convolution layers for image processing, w5 \& w0 are the weights of the classification of neural networks.

Step 7: Create a file to load the image and check for error in loading the images. 
Step 8: Create a file to train the convolutional layers. In this y1 stores the values of convolutional layer, y2 stores the ReLu value, y3 stores the pool value, y4 stores the value of reshaped size and y stores the softmax value.

Step 9: Create a random function rng for random values.

Step 10: Create a file to test the images. In this file, the images and labels are loaded for w1, w5 \& w0, when the images are constructed using CNN algorithm. Then finally, it will recognize the image by the classified image and feature learning.

\section{Sample Selection}

In our proposed work, the AR and ORL data samples are used since both the data sets are consisting the images that are mainly affected by some unconfined situations such as partially covered (wearing masks and sunglasses), various lighting images and various facial expressions that gives changes in the primary key factors of the face such as eyes, nose, mouth and cheeks. AR face data set consists of 100 individuals face images where 50 are of men and 50 are of women with ten different images for each individual. The ORL data set contains 400 pictures sized 112x92. This data set images are also a sample of images that are affected by unconfined situations. The 400 images are combination of men and women where 10 images are stored for each individual. The following fig shows the sample images of AR and ORL data sets.

\section{FINDINGS AND DISCUSSIONS}

\section{Accuracy and Computation Time}

In the proposed work, the experimental analysis is done with AR and ORL database. The proposed EVB_CNN is compared with the other face recognition algorithms such as NN and DBN for accuracy analysis and also for computation time where the proposed algorithm is shown with the $95 \%$ of accuracy which is significantly better than the others.

Table 1 shows the comparison of various face recognition algorithms. The NN algorithm shows $86 \%$, DBN algorithm shows $92 \%$ and our proposed algorithm EVB_CNN shows $95 \%$ that is comparably better than the other two algorithms.

\section{Error Rates}

The error rate is defined as the calculation of the frequently occurring errors. It is also defined as the ration of how much images are correctly recognized over with sent images. The error rates are

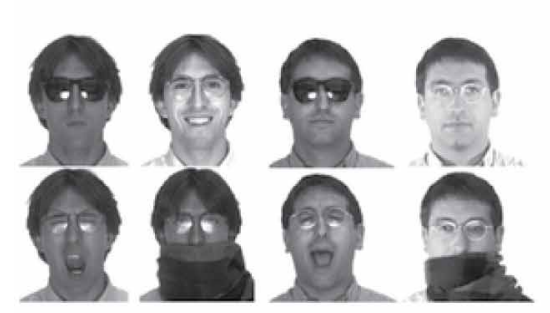

(a)

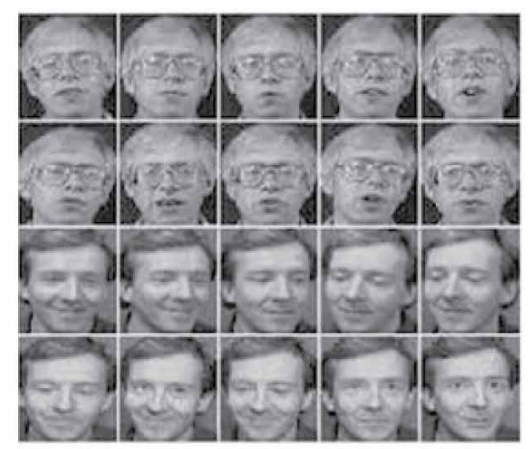

(b) 
Table 1. Comparison of various face recognition methods

\begin{tabular}{|l|l|}
\hline \multicolumn{1}{|c|}{ Algorithm } & \multicolumn{1}{c|}{ Recognition Accuracy } \\
\hline NN & $86 \%$ \\
\hline DBN & $92 \%$ \\
\hline Proposed EVB_CNN & $95 \%$ \\
\hline
\end{tabular}

analysed with different epoch values, time, minimum batch mean square value and full batch training error rates.

The Table 2 implies the set 1 error rate values for various epochs. For the epoch number $1 / 5$ the time is 3.1766 seconds, the minimum batch mean squared error on training set value is 0.16185 and the full batch training error rate is 0.0727 but for the epoch number $5 / 5$, all the values are decreased such as time is 2.1713 seconds, minimum batch mean squared error on training set is 0.11456 and the full batch training error rate is 0.0622 .

The set 2 error rates are even more reduced when compared to the set 1 error rates. The table 2 describes the set 2 error rates. For the epoch number $1 / 5$ the time is 1.1241 seconds, the minimum batch mean squared error on training set value is 0.9445 and the full batch training error rate is 0.38749 but for the epoch number $5 / 5$, all the values are decreased such as time is 1.1244 seconds, minimum batch mean squared error on training set is 0.2553 and the full batch training error rate is 0.23419 .

\section{Misclassification Error Rate}

It is the ratio of training or recall and testing or generalization of images from the given data set. It is the significant way of measuring the picture effectiveness in Neural networks. It is calculated as like error rate mentioned in the above section, but finally error mean square value is validated.

\section{Table 2. Set 1 Error rates}

\begin{tabular}{|l|l|l|l|}
\hline \multicolumn{1}{|c|}{ Epoch number } & \multicolumn{1}{|c|}{ Time(s) } & \multicolumn{1}{|c|}{$\begin{array}{c}\text { Minimum batch mean squared } \\
\text { error on training set }\end{array}$} & \multicolumn{1}{|c|}{$\begin{array}{c}\text { Full batch training } \\
\text { error rate }\end{array}$} \\
\hline $1 / 5$ & 3.1766 & 0.16185 & 0.0727 \\
\hline $2 / 5$ & 2.7505 & 0.15696 & 0.0626 \\
\hline $3 / 5$ & 2.5701 & 0.21087 & 0.0700 \\
\hline $4 / 5$ & 2.3612 & 0.12785 & 0.0673 \\
\hline $5 / 5$ & 2.1713 & 0.11456 & 0.0622 \\
\hline
\end{tabular}

Table 3. Set 2 Error rates

\begin{tabular}{|l|l|l|l|}
\hline \multicolumn{1}{|c|}{ Epoch number } & \multicolumn{1}{|c|}{ Time(s) } & $\begin{array}{c}\text { Minimum batch mean squared } \\
\text { error on training set }\end{array}$ & $\begin{array}{c}\text { Full batch training error } \\
\text { rate }\end{array}$ \\
\hline $1 / 5$ & 1.1241 & 0.9445 & 0.38749 \\
\hline $2 / 5$ & 1.1317 & 0.3599 & 0.31021 \\
\hline $3 / 5$ & 1.1410 & 0.3062 & 0.27994 \\
\hline $4 / 5$ & 1.1248 & 0.2759 & 0.24955 \\
\hline $5 / 5$ & 1.1244 & 0.2553 & 0.23419 \\
\hline
\end{tabular}


Table 4 describes about the Misclassification error rate along with mean square validation. For the epoch number $1 / 5$ the time is 1.6169 seconds, the minimum batch mean squared error on training set value is 1.0475 and the full batch training error rate is 0.40563 and validation of mean squared error rate is 0.44714 and for the epoch number 5/5, all the values are decreased such as time is 0.9264 seconds, minimum batch mean squared error on training set is 0.2614 , the full batch training error rate is 0.23667 and the validation of mean square error rate is 0.32153 . This analysis shows the improved performance of the proposed algorithm.

The graphical analysis of error and misclassification rate is done by considering the training and validation set of samples. The figure 6 describes about the Error and Misclassification rate.

The error rate and misclassification rate are plotted for training and validation phases consequently by considering the number of epochs and error rate. The validation samples are showing better performance than the training samples. The above analysis clearly mentioning the enhanced performance of the proposed algorithm that is used to overcome the problems occurred during the unconfined situations.

Table 4. Misclassification Error rate

\begin{tabular}{|l|l|l|l|l|}
\hline \multicolumn{1}{|c|}{ Epoch number } & \multicolumn{1}{|c|}{ Time(s) } & $\begin{array}{c}\text { Minimum batch mean } \\
\text { squared error on training } \\
\text { set }\end{array}$ & $\begin{array}{c}\text { Full batch training } \\
\text { error rate }\end{array}$ & $\begin{array}{c}\text { Validation Mean } \\
\text { squared error rate }\end{array}$ \\
\hline $1 / 5$ & 1.6169 & 1.0475 & 0.40563 & 0.44714 \\
\hline $2 / 5$ & 1.2199 & 0.3749 & 0.31722 & 0.36746 \\
\hline $3 / 5$ & 1.1146 & 0.3149 & 0.27946 & 0.34074 \\
\hline $4 / 5$ & 0.9313 & 0.2819 & 0.25261 & 0.32820 \\
\hline $5 / 5$ & 0.9264 & 0.2614 & 0.23667 & 0.32153 \\
\hline
\end{tabular}

Figure 6. Error rate and Misclassification rate
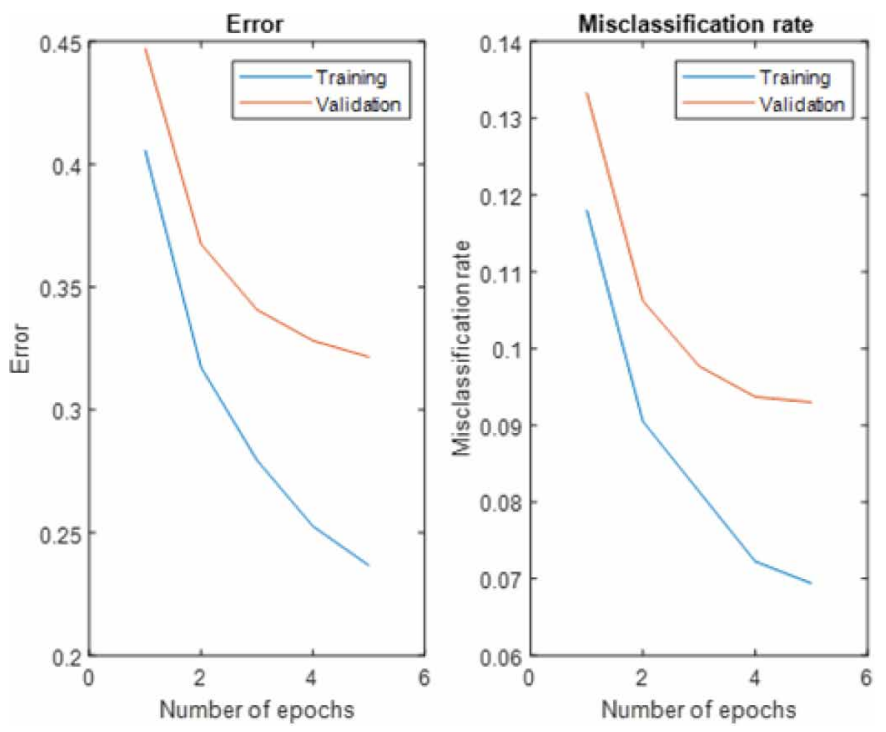


\section{CONCLUSION AND RECOMMENDATIONS}

Face recognition facing many challenging issues even though number of algorithms are in practise which are performing poor under some unconfined situations such as moving picture processing, poor lighting conditions, resolution less images and also for the positional change images. Hence in our work, a new hybrid framework EVB_CNN (Eigen Value Based Convolutional Neural Network) using tensor flow is proposed that ensures an enhanced performance in the recognition of images in an effective manner. In this proposed method, first the image is resized and then the Eigen values are calculated and finally converted to matrices before trained by the CNN. From the calculated Eigen values, the face region and non-face region is detected and the non-face regions are removed and hence the dimension is reduced that has been trained by the CNN. These resized samples are processed through layer by layer in proposed CNN model where in convolution layer the matrices structure is further reduced in dimension and based on the feature maps are created. The feature maps are then processed in activation layer and finally sent to the softmax layer for efficient classification. The face is recognized through classification and feature learning by the various layers present in the CNN model. This shows the face recognition accuracy rate of $95 \%$ which is significantly better than other algorithms. For various epoch sets the error and misclassification rate are analysed. The resizing is done in the pre-processing stage and also in the eigen value calculation stage so that the samples that are trained by $\mathrm{CNN}$ are reduced in dimension which allows to process more samples and also reducing the computation time. The accuracy can be improved even more if the same set of samples are trained by CNN for the consecutive times. The proposed hybrid frame work is struggling to recognize the occluded images in an effective manner. Hence, in future work, any other algorithm can be integrated to recognize the occluded images well with huge sample size with less computation. 


\section{REFERENCES}

Behera, A., Gidney, A., Wharton, Z., Robinson, D., \& Quinn, K. (2019). A CNN Model for Head Pose Recognition using Wholes and Regions. .10.1109/FG.2019.8756536

Coşkun. (2017). Face Recognition Based on Convolutional Neural Network. doi:10.1109/MEES.2017.8248937

Dong, J., \& Zheng, H., \& Lian, L. (2018). Dynamic Facial Expression Recognition Based on Convolutional Neural Networks with Dense Connections. 10.1109/ICPR.2018.8545596

Elmahmudi, A., \& Ugail, H. (2019). Deep face recognition using imperfect facial data. Future Generation Computer Systems, 99, 213-225. Advance online publication. doi:10.1016/j.future.2019.04.025

Hien, D., Sagayam, M., Bruntha, M., Subramaniyam, D., Amir, A., Jone, A., \& Ganesan, R. (2019). Image Fusion based on Sparse Sampling Method and Hybrid Discrete Cosine Transformation. International Journal of Scientific \& Technology Research, 8, 1103-1107.

Kim, Kim, Roy, \& Jeong. (2017). Efficient Facial Expression Recognition Algorithm Based on Hierarchical Deep Neural Network Structure. IEEE Access. 10.1109/ACCESS.2017.DOI

Koo, J. H., Cho, S. W., Baek, N. R., Kim, M. C., \& Park, K. R. (2018). CNN-Based Multimodal Human Recognition in Surveillance Environments. Sensors (Basel), 18(9), 3040. doi:10.3390/s18093040 PMID:30208648

Li, J., Qiu, T., Wen, C., Xie, K., \& Wen, F. (2018). Robust Face Recognition Using the Deep C2D-CNN Model Based on Decision-Level Fusion. Sensors (Basel), 18(7), 2080. doi:10.3390/s18072080 PMID:29958478

Rajesh, G., Mercilin Raajini, X., Martin Sagayam, K., Bhushan, B., \& Utku, K. (2020). Fuzzy genetic based dynamic spectrum allocation (FGDSA) approach for cognitive radio sensor networks. Turkish Journal of Electrical Engineering and Computer Sciences.

Ramesh Babu \& Reddy. (2019). A Design Of Eigenvalue Based CNN Tool For Image Retrieval. International Journal of Engineering and Advanced Technology, 8(6).

Ramkumar, G., \& Logashanmugam, E. (2018). Hybrid framework for detection of human face based on haarlike feature. IACSIT International Journal of Engineering and Technology, 7(3), 1786-1790. doi:10.14419/ijet. v7i3.16227

Shen, Y., Han, T., Yang, Q., Wang, Y., Li, F., \& Wen, H. (2018). CS-CNN: Enabling Robust and Efficient Convolutional Neural Networks Inference for Internet-of-Things Applications. IEEE Access : Practical Innovations, Open Solutions. Advance online publication. doi:10.1109/ACCESS.2018.2810264

Syazana-Itqan, K., Syafeeza, A. R., \& Saad, N. M. (2016). A MATLAB- Based Convolutional Neural Network Approach for Face Recognition System. Bioinfo Proteom Img Anal, 2(1), 71-75.

Tamilselvi, M., \& Karthikeyan, S. (2019). A Face Recognition System using Directional Binary Code Algorithm and Multi-SVM. International Journal of Engineering and Advanced Technology, 8(6S3).

Tian, H., Cai, H., Lai, J.-H., \& Xu, X. (2011). Effective image noise removal based on difference eigenvalue. Proceedings - International Conference on Image Processing, ICIP, 3357-3360. doi:10.1109/ICIP.2011.6116392

Wang, Z., Wang, Z., Zhang, H., \& Guo, X. (2017). A Novel Fire Detection Approach Based on CNN-SVM Using Tensorflow. .10.1007/978-3-319-63315-2_60

Yan, K., Huang, S., Song, Y., Liu, W., \& Fan, N. (2017). Face recognition based on convolution neural network. 2017 36th Chinese Control Conference (CCC), 4077-4081.

Yang, X., \& Li, M. (2017). Facial Expression Recognition Algorithm Based on CNN and LBP Feature Fusion. doi:10.1145/3175603.3175615

Yuan, L., Qu, Z., Zhao, Y., Zhang, H., \& Nian, Q. (2017). A convolutional neural network based on TensorFlow for face recognition. 10.1109/IAEAC.2017.8054070

Zhang, M., Liang, Y., \& Ma, H. (2019). Context-Aware Affective Graph Reasoning for Emotion Recognition. 10.1109/ICME.2019.00034 
M. Tamilselvi is Research scholar from Dept of ECE at Sathyabama Institute of science and technology, Chennai, India. She completed her UG in ECE in the year 2005 from C.Abdul Hakkem College of Engineering and Technology, Vellore and received her M. Tech in VLSI Design from Sathyabama University in 2013. She is having more than 10 years of teaching experience in various engineering colleges and published many research papers in National and International journals and conferences.

S. Karthikeyan (PhD) was born in 1979. He received his Bachelor's degree in Electronics and Instrumentation Engineering from Annamalai University. He received a Master's degree in Applied Electronics from Sathyabama University, Chennai. He received Ph.D in Wireless sensor Network from Sathyabama University. He is a Fellow in "The institution of engineers(India)". He is currently working as an Associate Professor in the Department of Electronics and Communication Engineering, besides coordinator, International Affairs school of electrical and electronics engineering. Sathyabama Institute of science and technology, Chennai. His areas of interest include Mobile Agent, Wireless Sensor Networks, Wireless Communication, Image Processing, Signal Processing, IoT and Cryptography. He has about 18 years of experience in teaching. Board of studies Member in Vel's university, Chennai. He has published 45 papers in International / National Journals/ conferences. He was a reviewer in a top Journals / conferences like Elsevier, Inderscience, etc., He guided 20 PG students in India and a PG student in rostock university, Germany. Doctoral committee member in Anna university, Vel's institute and veltech institute. Nodal Incharge for CDAC PG courses in Sathyabama. Active involvement in MOU with industrial. 